Flávia de Araújo Berenguer ${ }^{1}$

Dayse de Amorim Lins e Silva ${ }^{2}$

Celina Cordeiro de Carvalho ${ }^{3}$

'Fisioterapeuta, Especialista em Fisioterapia do Trabalho com Ênfase em Ergonomia, Especialista em Fisioterapia Vascular, Professora da Faculdade Maurício de Nassau, Recife, PE, Brasil.

${ }^{2}$ Fisioterapeuta, Mestre em Biofísica, Professora da Faculdade Integrada do Recife, Recife, PE, Brasil.

${ }^{3}$ Fisioterapeuta, Doutora em Neurociências pela Universidade Federal de Pernambuco, Professora da Faculdade Integrada do Recife, Recife, PE, Brasil.

*Artigo baseado no trabalho de conclusão do curso de pós-graduação em Fisioterapia do Trabalho de Flávia de Araújo Berenguer, apresentado em 2008 à Faculdade Redentor, Rio de Janeiro, RJ.

Contato:

Flávia de Araújo Berenguer

Estrada das Ubaias, ㄲo 75 apt. 401

CEP: 52070-013 - Recife-PE

E-mail:

flavia_berenguer@yahoo.com.br

Recebido: 25/03/2010

Revisado: $11 / 12 / 2010$

Aprovado: 23/12/2010

\section{Influência da posição ortostática na ocorrência de sintomas e sinais clínicos de venopatias de membros inferiores em trabalhadores de uma gráfica na cidade do Recife-PE*}

\author{
Influence of orthostatic posture in the occurrence of clinical \\ symptoms and signs of lower limb venopathy in workers of a \\ printing company in Recife, Pernambuco, Brazil
}

\section{Resumo}

Embora ainda não haja evidência da relação direta causa-efeito da doença venosa com o trabalho, existe consenso atual na opinião médica de que a postura de trabalho pode agravar o desenvolvimento da mesma. O presente estudo teve como objetivo avaliar a influência da permanência prolongada na postura ortostática com o desencadeamento e/ou agravamento de sinais e sintomas referentes aos transtornos venosos de membros inferiores em funcionários de uma gráfica em Recife, entre maio e julho de 2008. Foram analisadas as condições e os meios que são colocados à disposição do trabalhador para desenvolver sua atividade laboral. Os funcionários responderam a um questionário onde foram abordadas questões referentes aos transtornos circulatórios de membros inferiores com queixas ao longo do dia e no final do expediente de trabalho. Também foi realizada a perimetria dos membros inferiores antes e após a jornada de trabalho. Foi observada a presença de varizes em $64 \%$ dos funcionários e o aumento das medidas de perimetria em todos os funcionários após a jornada de trabalho. Dentre as queixas relatadas, destaca-se a sensação de peso e cansaço, dores, cãimbras e presença de edema nos membros inferiores. Os resultados indicam uma associação positiva entre as condições de trabalho no setor investigado e o surgimento ou agravamento de sinais e sintomas referentes aos transtornos venosos nos membros inferiores nos funcionários.

Palavras-chaves: doenças ocupacionais; insuficiência venosa; ambiente de trabalho.

\begin{abstract}
Although there is no evidence of direct causal relationship between venous disease and work, currently there is consensus of medical opinion that posture at work can aggravate this type of disease. The purpose of this study was to evaluate the influence of prolonged orthostatic posture in the onset and/or worsening of signs and symptoms related to lower limb venous disorders among employees of a printing company in Recife, between May and July 2008. We analyzed the conditions and means made available for employees to perform their labor activities. They answered a questionnaire with questions related to lower limb circulatory disorders, reporting complaints throughout and at the end of working day. Lower limbs perimetry was tested before and after work. Sixty-four per cent of workers had varicose veins and increased perimetry measures after their working hours. Reported complaints included feeling heavy, fatigue, pains, cramps, and lower limb edema. The results indicated a positive association between working conditions at the investigated company and emerging or worsening of signs and symptoms related to venous disorders in lower limbs of its employees.
\end{abstract}

Keyswords: occupational diseases; venous insufficiency; work environment. 


\section{Introdução}

O estudo das condições de saúde e trabalho de grupos ocupacionais permite caracterizar os processos laborais e descrever o perfil de adoecimento dos trabalhadores, avaliando possíveis associações entre ocupação e saúde (ARAÚJO et al., 2005). A literatura sugere que muitos problemas de saúde ocupacional estão associados com a permanência da postura em pé e inúmeras são as situações de trabalho que requerem a manutenção desta postura por um longo período de tempo, sendo adotada em vários postos de trabalho da indústria e do comércio (GRANDJEAN, 1998; RENNER, 2002; JORGE, 2003; JÚNIOR, 2004; GUIMARÃES, 2006; LUZ, 2006).

A postura parada em pé exige o trabalho estático da musculatura envolvida para manutenção dessa posição, provocando facilmente a fadiga muscular (GUIMARÃES, 2006). Autores como Renner (2002), Jorge (2003) e Júnior (2004) sugerem que a postura em pé, além de ser causa direta de fadiga, também é causa de dores e desconfortos nas costas e nos membros inferiores. A fadiga e o desconforto, mesmo que não levem a uma incapacidade, podem diminuir a resistência dos trabalhadores, levando-os a adquirir doenças e até mesmo sintomas de origem ocupacional, como varicoses (ZHANG; DRURY; WOLLEY, 1991).

Para a manutenção da postura estática em pé, são necessários níveis baixos, porém constantes, de tensão muscular, e esse estado prolongado de contração provoca uma compressão dos vasos sanguíneos (GRANDJEAN, 1998) prejudicando a circulação sanguínea e linfática. Como consequência, pode-se observar o aparecimento de alguns transtornos circulatórios nos membros inferiores, como varizes, edema e celulite (LUZ, 2006). Estes transtornos, além do comprometimento estético e funcional, comumente desencadeiam dores e parestesias, podendo evoluir para a perda parcial ou total da mobilidade dos membros inferiores (LUZ, 2006).

Algumas categorias profissionais são fatores predisponentes para o aparecimento ou a piora da síndrome varicosa, sobretudo aquelas que exigem posição ortostática prolongada (SILVA, 2002).

A doença venosa é um problema de saúde pública importante, pois acomete pessoas de diferentes faixas etárias e pode causar sérios problemas socioeconômicos, como, por exemplo, a inaptidão para o trabalho, pois apresenta uma repercussão indireta sobre a qualidade da produção e consequente perda de eficiência operacional. É responsável por absenteísmos e hospitalizações e até mesmo por aposentadoria de indivíduos na fase produtiva da vida (FRANÇA; TAVARES, 2003; LUZ, 2006).

As venopatias apresentam elevada incidência e prevalência, representam um quadro clínico comum a várias afecções que têm como base o comprometimento crônico do retorno venoso, sendo associadas com varizes, edemas, dores e desconfortos nos membros inferiores (MELLO, 1999; FRANÇA; TAVARES, 2003). O edema é um sinal precoce das venopatias crônicas e uma das formas normalmente utilizadas para sua quantificação é a perimetria, que consiste na mensuração da circunferência dos membros inferiores (PERRIN; GUEX, 2000).

A manutenção da postura em pé é muitas vezes relacionada a varizes de membros inferiores (MELLO, 1999; LUZ, 2006). Para Renner (2002), o papel da postura durante o trabalho no aumento das varizes é uma questão ainda muito controversa, já para Silva (1999) a circulação venosa periférica é profundamente afetada pela postura. Segundo Monteiro e Bertagni, citados por Luz (2006), embora não haja evidência da relação direta causa-efeito de doença venosa com o trabalho, existe consenso atual na opinião médica de que o trabalho pode agravar seriamente o desenvolvimento da mesma.

Dada a sua importância, e diante da falta de informações mais concretas, faz-se necessária uma caracterização mais específica da relação entre o desenvolvimento ou agravamento de doenças venosas nos membros inferiores e a posição ortostática no trabalho. Dessa forma, este estudo utiliza o enfoque da ergonomia, uma abordagem que analisa a relação entre o homem e o trabalho, para avaliar a influência da permanência prolongada na postura ortostática com o desencadeamento e/ou agravamento de sinais e sintomas referentes aos transtornos venosos de membros inferiores em funcionários que trabalham de pé em uma gráfica na cidade do Recife-PE.

\section{Metodologia}

O presente estudo foi desenvolvido com os funcionários do setor de acabamento de uma gráfica da Região Metropolitana do Recife-PE, no período de maio a julho de 2008. Foram convidados a participar da pesquisa aqueles que trabalhavam diariamente no setor de acabamento e que exerciam sua atividade laboral na posição ortostática. Eles também deveriam ter no mínimo um ano de atividade no setor.

Foram considerados como critérios de exclusão os funcionários que trabalhassem em postura predominantemente sentada e as funcionárias que estivessem grávidas. A fim de evitar viés, misturando indivíduos que realizavam tratamento profilático com os que não realizavam, também foram excluídos os funcionários que faziam uso de meia elástica, visto que o estudo tinha o propósito de avaliar as alterações causadas pela postura em pé e não identificar quem já tinha alguma patologia venosa.

Dos 70 funcionários que trabalhavam no setor de acabamento da gráfica estudada, apenas 53 participaram do nosso estudo, pois 17 deles foram excluídos: 2 exerciam sua atividade laboral em posição predominantemente sentada, 8 trabalhavam a menos de um ano no setor, 3 estavam gestantes e 4 faziam uso de meias elásticas.

Foi realizada uma observação direta do trabalho no setor de acabamento da gráfica, onde foram analisadas as condições e os meios colocados à disposição dos trabalhadores para desenvolverem suas atividades laborais que pudessem interferir no desenvolvimento ou agravamento de doenças venosas de membros inferiores. 
Os funcionários do setor de acabamento que se dispuseram a participar da pesquisa responderam a um questionário, o qual foi elaborado tendo como base o utilizado por Luz (2006). Foram abordadas questões relacionadas ao estado de saúde geral dos indivíduos, enfatizando as questões referentes aos transtornos circulatórios de membros inferiores (varizes, ocorrência de dor, sensação de peso ou cansaço, cãimbras e edema, entre outros) com queixas ao longo do dia e no final do expediente de trabalho. Além disso, foram questionados: idade, sexo, peso, altura, carga horária de trabalho, tempo de serviço no setor e os antecedentes pessoais e familiares do trabalhador, a fim de permitir uma abordagem ampla às condições clínicas, assim como a identificação dos fatores de risco.

Em seguida, os participantes foram submetidos à perimetria dos membros inferiores antes e após a jornada de um dia de trabalho, a qual foi realizada no ambulatório médico da empresa. A perimetria é um método de avaliação facilmente aplicado na clínica, sendo adotada neste estudo por melhor se adequar às condições existentes na empresa avaliada. As mensurações foram realizadas, segundo Perrin e Guex (2000), com o funcionário na posição sentada com as pernas pendentes, em centímetros, e com auxílio de uma fita métrica. Foram estipulados três pontos: circunferência maior da panturrilha, tornozelo e pé, tendo, como ponto de referência, o maléolo lateral. Os pontos foram marcados com auxílio da caneta dermográfica para que as mensurações fossem realizadas exatamente na mesma altura antes e depois da jornada de trabalho e assim ter um valor comparativo.

Os resultados são apresentados sob a forma de média \pm desvio padrão (DP) para as variáveis com distribuição normal. A normalidade e a homogeneidade dos valores mensurados foram determinadas pelo teste de Kolmogorov-Smirnov e Levene, respectivamente. Para as comparações entre as avaliações do mesmo grupo foi utilizado o teste t-student para amostra pareada e, para comparar cada avaliação entre os grupos, o teste $t$-student para amostras independentes. O nível de significância adotado foi de 5\%, sendo os dados analisados através do programa estatístico SPSS para Windows versão 16.0.

O estudo foi aprovado pelo Comitê de Ética em Pesquisa do Hospital Agamenon Magalhães - Secretaria de Saúde do Estado de Pernambuco, com parecer $\mathrm{n}^{\circ}$ 262/2008. Todos os voluntários foram esclarecidos sobre os objetivos e procedimentos do estudo e assinaram um termo de consentimento livre e esclarecido conforme as normas para pesquisa envolvendo seres humanos, resolução 196/96 do Conselho Nacional de Saúde.

\section{Resultados}

Foram avaliadas 53 funcionárias do setor de acabamento da gráfica estudada que trabalhavam de segunda a sábado divididas em dois turnos de trabalho: das 6 horas às 14 horas e das 14 horas às 22 horas.

Durante todo o ano, há um controle rígido da produtividade na gráfica, no entanto, nos meses que antecedem o período escolar (setembro a fevereiro), a demanda da produção é muito maior e as funcionárias são submetidas a um aumento da carga horária diária (hora extra), chegando a trabalhar algumas vezes de domingo a domingo. Dentro da sistemática organizacional da empresa, as trabalhadoras fazem algumas pausas já prescritas: diariamente, há uma pausa para o lanche e uma para o almoço/jantar e, duas vezes na semana, fazem pausa para a ginástica laboral.

O trabalho realizado no setor de acabamento é exclusivamente manual, no entanto, as funcionárias passam em média 6 horas na posição ortostática desenvolvendo suas atividades laborais, pois neste setor os postos de trabalho não são adaptados para alternância de postura nem apresentam assentos para descanso. Foi observado que na parte inferior da bancada de trabalho existe uma estrutura metálica, onde, eventualmente, as funcionárias apoiam um dos pés para aliviar o desconforto nos membros inferiores.

Sobre as características da amostra, todos os participantes do estudo eram do sexo feminino, com idade média de 29,2 anos $( \pm 5,33)$, e apresentavam, em média, 4,47 anos $( \pm 2,36)$ de tempo de serviço no setor. Nenhuma referiu outro vínculo empregatício, no entanto, todas relataram que executam atividades domésticas diariamente em suas residências. Ao responderem o questionário, nenhuma funcionária relatou ser diabética, no entanto, 2 alegaram hipertensão arterial; 34, varizes de membros inferiores; e 9 relatarem outras doenças. Do total de funcionárias investigadas, apenas 12 faziam uso de medicamentos regularmente, sendo 2 de anti-hipertensivos e 10 de contraceptivos orais. Apenas 12 funcionárias nunca ficaram grávidas, no entanto, 10 tiveram apenas uma gestação, 23, duas gestações e 8 mais de três gestações. Em relação aos hábitos de vida, apenas 3 funcionárias praticavam atividades físicas regulares, 4 eram fumantes e 3 ex-fumantes, 16 relataram etilismo e 11 faziam uma dieta balanceada. Em relação ao índice de massa corpórea (IMC), apenas 28 das funcionárias participantes do estudo apresentaram peso normal (Tabela 1).

A postura predominante de trabalho considerada por todas as funcionárias foi a parada em pé (ortostática). O carregamento de peso também foi relatado como uma constante por todas as funcionárias, destacando-se o levantamento e o transporte manual dos livros dos paletes para a bancada de trabalho, e viceversa. $\mathrm{O}$ apoio de peso predominante no membro inferior direito foi relatado por 5 funcionárias (9,43\%), no membro inferior esquerdo por $3(5,66 \%)$ e alternando entre os dois por 45 funcionárias (84,91\%).

Em relação às doenças venosas, 34 funcionárias $(64,15 \%)$ relataram presença de varizes nos membros inferiores, destas, 14 apresentavam antecedentes familiares. Nenhuma das funcionárias alegou ter feito tratamento específico para venopatias. Com relação às questões objetivas específicas, quanto à ocorrência de queixas relacionadas aos transtornos circulatórios de membros inferiores, os dados referentes podem ser visualizados na Tabela 2. 
Tabela 1 Índice de massa corpórea (IMC) das funcionárias incluídas no estudo

\begin{tabular}{lcc}
\hline \multicolumn{1}{c}{ IMC } & $n^{\text {o de funcionários }}$ & $\%$ \\
\hline Baixo peso & 4 & 8 \\
Normal & 28 & 53 \\
Sobrepeso & 19 & 36 \\
Obeso & 2 & 4 \\
Obeso grave & 0 & 0 \\
Obeso mórbido & 0 & 0 \\
\hline
\end{tabular}

Tabela 2 Queixas referidas pelas funcionárias incluídas no estudo relacionadas aos transtornos circulatórios nos membros inferiores

\begin{tabular}{lc}
\hline \multicolumn{1}{c}{ Relatos } & $n^{\text {Oode funcionários }}$ \\
\hline Sensação de peso ou cansaço nas pernas & 39 \\
com frequência & 5 \\
algumas vezes & 9 \\
não ocorria & \\
Sensação de inchaço & 29 \\
com frequência & 11 \\
algumas vezes & 13 \\
não ocorria & \\
Dores ou cãimbras & 21 \\
com frequência & 22 \\
algumas vezes & 10 \\
não ocorria & \\
Elevação das pernas em casa & \\
com frequência & \\
algumas vezes & 22 \\
não ocorria & \\
\hline
\end{tabular}

Das 53 funcionárias avaliadas, 49 (92,45\%) consideram que suas queixas aumentam no decorrer do dia e apenas $4(7,54 \%)$ afirmaram que as mesmas não se alteram. Quando perguntadas se o fato de caminhar alterava essas queixas, 40 (75,47\%) disseram que diminuíam, 5 (9,43\%) que aumentavam e $8(15,1 \%)$ que não fazia diferença. Porém, todas foram unânimes em afirmar que permanecer na postura parada em pé, por períodos de tempo maiores, piorava os desconfortos relatados.

Todas as funcionárias, sem exceção, referiram acreditar que existe uma relação direta entre as queixas relatadas e as condições que executam o seu trabalho no setor de acabamento da gráfica. Apenas três funcionárias rela- taram apresentar as mesmas queixas (sensação de peso, cansaço, inchaço, dores ou cãimbras) nos dias de folga.

A avaliação através da perimetria mostrou que houve variação na circunferência de ambos os membros inferiores, em um dia de trabalho, em todas as funcionárias avaliadas, as quais foram mensuradas no início e ao término da jornada de trabalho (Tabela 3), principalmente na região do tornozelo, onde a diferença média da circunferência antes e após o trabalho foi de $0,3 \mathrm{~cm}$. No entanto, não houve diferença ao comparar as variações das medidas de perimetria entre as medidas dos segmentos dos membros inferiores direito e esquerdo, tanto antes como após a jornada de trabalho (Tabela 4). 
Tabela 3 Comparação das medidas (média \pm desvio padrão) de perimetria (cm) em cada segmento de membro inferior direito (MID) e esquerdo (MIE), antes e após a jornada de trabalho $(n=53)$

\begin{tabular}{clllc}
\hline & Região & Antes $(\mathrm{cm})$ & Após $(\mathrm{cm})$ & pvalor* $^{*}$ \\
\hline \multirow{3}{*}{ MID } & Panturrilha & $32,6 \pm 1,8$ & $32,8 \pm 1,9$ & 0,000 \\
& Tornozelo & $21,4 \pm 1,6$ & $21,7 \pm 1,7$ & 0,000 \\
& Pé & $20,2 \pm 1,3$ & $20,4 \pm 1,3$ & 0,000 \\
& Panturrilha & $32,6 \pm 1,8$ & $32,6 \pm 2,1$ & 1,0 \\
& Tornozelo & $21,3 \pm 1,6$ & $21,6 \pm 1,7$ & 0,000 \\
& Pé & $20,2 \pm 1,3$ & $20,4 \pm 1,3$ & 0,000 \\
\hline
\end{tabular}

* teste t-student para amostra pareada

Tabela 4 Comparação das medidas (média \pm desvio padrão) de perimetria $(\mathrm{cm})$ entre segmentos do membro inferior direito (MID) e esquerdo (MIE), antes e após a jornada de trabalho $(\mathrm{n}=53)$

\begin{tabular}{lcccccc}
\hline & \multicolumn{2}{c}{ Antes } & \multicolumn{3}{c}{ Após } \\
\multicolumn{1}{r}{ Região } & MID $(\mathrm{cm})$ & MIE $(\mathrm{cm})$ & p valor & MID $(\mathrm{cm})$ & MIE $(\mathrm{cm})$ & pvalor * $^{*}$ \\
\hline Panturrilha & $32,6 \pm 1,8$ & $32,6 \pm 1,8$ & 0,942 & $32,8 \pm 1,9$ & $32,6 \pm 2,1$ & 0,630 \\
Tornozelo & $21,4 \pm 1,6$ & $21,3 \pm 1,6$ & 0,906 & $21,7 \pm 1,7$ & $21,6 \pm 1,7$ & 0,816 \\
Pé & $20,2 \pm 1,3$ & $20,2 \pm 1,3$ & 0,916 & $20,4 \pm 1,3$ & $20,4 \pm 1,3$ & 0,839 \\
\hline
\end{tabular}

* teste t-student para amostra pareada

\section{Discussão}

Muitas situações de trabalho requerem a manutenção da postura em pé por um longo período de tempo (JORGE, 2003). Segundo Brasil (2002), a escolha desta postura só se justifica quando a tarefa exige deslocamentos contínuos, manipulação de cargas pesadas, alcances amplos frequentes, mudanças constantes de postos de trabalho e/ou aplicação de força para baixo. Essas condições de trabalho não foram observadas no setor de acabamento da gráfica, onde foi realizado o estudo, no entanto, as funcionárias passam, em média, 6 horas em ortostatismo desenvolvendo suas atividades laborais.

Segundo Ryan (1989), citado por Hansen, Winkel e Jorgensen (1998), a manutenção da postura em pé por $45 \%$ a $50 \%$ da carga horária de trabalho é suficiente para causar sintomas regulares de dores nas pernas e nos pés. No presente estudo, foi evidenciado que o tempo médio de permanência nessa postura era de 6 horas, correspondendo a $75 \%$ da carga horária diária de trabalho, e, corroborando com os autores citados, este valor representa um risco para o desenvolvimento de dor e desconforto nos membros inferiores, fato este confirmado com as respostas das funcionárias ao questionário.
A situação de trabalho analisada neste estudo expõe as funcionárias a uma jornada de trabalho extensa e com tempo reduzido de descanso; fatores estes que podem ter contribuído para o aparecimento e o agravamento da sintomatologia referentes a transtornos venosos de membros inferiores das funcionárias avaliadas, independentemente dos fatores de risco associados. Este achado está de acordo com Luz (2006), a qual considera que a manutenção na posição em pé, mantida por uma longa jornada de trabalho diária, pode desencadear vários problemas de saúde aos trabalhadores, entre os quais estão o maior desgaste físico e as queixas relacionadas aos transtornos circulatórios de membros inferiores, como dores, edema, sensação de peso, parestesia e cãimbras.

A manutenção da postura em pé imóvel apresenta várias desvantagens como: fadiga, dor, desconforto, tensão muscular e tendência à acumulação do sangue nas pernas, o que predispõe ao aparecimento de insuficiência valvular venosa nos membros inferiores, resultando em varizes e sensação de peso nas pernas (BRASIL, 2002). No presente estudo foi verificado que, das 53 funcionárias avaliadas, 39 (73,58\%) queixavamse frequentemente de sensação de peso nas pernas, 21 (39,62\%), de dor e 29 (54,71\%), de sensação de inchaço nas pernas (edema), além disso, 34 (64,15\%) apresentavam varizes. Resultados estes que corroboram Luz 
(2006), que realizou um estudo similar com 14 cozinheiras industriais e evidenciou que $92,85 \%$ relataram sensação de peso e dor nas pernas, $71,42 \%$, inchaço e $78,57 \%$ apresentavam varizes.

Segundo Pitta, Fonseca e Santos (2003), as varizes apresentam uma prevalência aproximada de 37,9\% na população geral. Maffei e colaboradores (1986), citados por Silva (2002), também estimaram uma prevalência de varizes de $35,5 \%$ ao realizarem um estudo epidemiológico de alterações venosas de membros inferiores da população de Botucatu-SP. No presente estudo, foi verificado que $64,15 \%$ das funcionárias apresentavam varizes de membros inferiores, o que leva a crer que a postura ortostática adotada durante a atividade laboral pode ter influenciado no aumento dessa prevalência. Além disso, acrescenta-se o fato de que a média de idade das funcionárias avaliadas foi de 29,2 anos e, segundo Azizi (2001) e Silva (2002), a incidência de varizes é mais alta a partir da terceira década de vida.

As funcionárias participantes deste estudo trabalham em um setor onde há um controle rígido da produtividade durante todo o ano, o qual aumenta quando a demanda da produção é maior, sendo as funcionárias submetidas a horas extras, permanecendo mais tempo no local de trabalho e aumentando ainda mais o tempo de exposição à carga estática. Para Dejours (1992) e Jorge (2003), muitas vezes a rigidez operativa de trabalho com contratempos organizacionais levam ao excesso de carga física e psicológica, principalmente em uma atividade profissional onde são exigidas do trabalhador metas de produção, tornando-o mais suscetível a doenças. Fato este confirmado neste estudo, em que as funcionárias foram unânimes em afirmar que permanecer na postura parada em pé por períodos de tempo maiores, como ocorre quando são submetidas a horas extras, piora os desconfortos relatados.

Algumas categorias profissionais são consideradas como fatores predisponentes para o aparecimento, ou a piora, da doença venosa, sobretudo aquelas que exigem o uso da posição ortostática prolongada (SILVA, 2002), como é o caso da situação de trabalho avaliada neste estudo. A profissão é um dado importante a se considerar, no entanto, não se podem desconsiderar os fatores de riscos associados à postura de trabalho, como a hereditariedade, a idade, o sexo, o número de gestações, a obesidade, a dieta, as patologias associadas, o uso de determinados medicamentos, o sedentarismo, entre outros (PARISELLE et al., 1992; KRIJNEN et al., 1997; BAREL; MATSUI, 1997; MELLO, 1999; SILVA, 2002).

Pariselle et al. (1992), ao analisar 2.985 prontuários de trabalhadores parisienses de diferentes categorias profissionais, não evidenciaram relação direta entre os sinais clínicos de doença venosa considerando os antecedentes familiares e a postura adotada durante a atividade laboral. No presente estudo, das 34 funcionárias que relataram presença de varizes nos membros inferiores, apenas $42 \%$ apresentavam antecedentes familiares, desta forma, corroborando a pesquisa de Pariselle (1992), a hereditariedade não apresentou relação direta para a ocorrência de sinais clínicos de venopatia.
Para Allaert, Levardo e Vin (1991), vários fatores podem favorecer a doença venosa quando associados ao sobrepeso e à obesidade, como o sedentarismo e o número de paridades. Das 53 funcionárias avaliadas no presente estudo, 21 apresentaram IMC acima do normal; destas, apenas 2 nunca tiveram filhos e nenhuma delas praticava atividades físicas regulares. Dentro do contexto analisado, estes achados podem potencializar vários outros elementos que favorecem a doença venosa, como a própria manutenção da postura em pé.

O número de gestações também apresenta influência no surgimento ou agravamento de doenças venosas (ALLAERT; LEVARDON; VIN, 1991; KRIJNEN et al., 1997; MELLO, 1999; SILVA, 2002). A hipótese é de que a gravidez acarrete alterações hormonais, aumento de volume sanguíneo e obstrução do retorno venoso, constituindo algumas possíveis causas de desencadeamento da doença (KRIJNEN et al., 1997). Foi observado que 41 das funcionárias $(77,35 \%)$ avaliadas já tiveram gestações, o que pode ter influenciado no número de queixas relativas ao sistema venoso. No entanto, as funcionárias avaliadas que nunca ficaram grávidas também se queixaram de dores, edema, cãimbras, sensação de peso e cansaço nos membros inferiores, fatores estes que apresentam maior relação com as condições de trabalho.

Segundo Pariselle et al. (1992) e Krijnen et al. (1997), a utilização de hormônios exógenos tem relação direta com a etiopatogenia das varizes primárias. No entanto, das 10 funcionárias avaliadas que faziam uso de contraceptivo oral, apenas 4 relataram presença de microvarizes, dessa forma não se pode considerar a utilização de hormônios como fator isolado para a ocorrência de varizes, visto que, de acordo com os autores citados, teoricamente, todas as 10 funcionárias deveriam apresentar queixas de se varizes. Dessa forma, percebe-se a importância de se associar a postura de trabalho com os outros fatores de risco.

Quanto ao sexo, segundo Silva (2002), a maior incidência das varizes é entre as mulheres devido aos fatores hormonais. No entanto, não foi possível confirmar este fato, pois a população do presente estudo era formada apenas por mulheres.

As queixas das funcionárias avaliadas com relação às doenças venosas foram descritas como sensação de peso e cansaço (83\%), edema (75,47\%), dor e cãimbras nas pernas $(81,13 \%)$, as quais aumentam ao longo do dia, especialmente após a permanência prolongada da postura em pé. Estes achados são semelhantes aos referidos por Luz (2006), em seu estudo com cozinheiras, no qual 92\% queixaram-se de sensação de peso e cansaço, dor e cãimbra e 71,42\% de presença de edema.

Segundo Mello (1999), quando o indivíduo fica muito tempo em pé, parado, os sintomas tendem a se agravar, este fato também foi observado neste estudo, em que 49 das funcionárias avaliadas (92,45\%) consideram que suas queixas aumentam no decorrer do dia. Luz (2006) relata em seu estudo que 92,85\% também se queixavam do aumento dos sintomas quando passavam muito tempo em pé. 
Os sintomas nos membros inferiores em geral são aliviados após repouso com os membros elevados (MELLO, 1999; CRAAGER; DZAU, 2002). Apesar das funcionárias avaliadas possuírem este conhecimento, apenas quatro delas $(7,54 \%)$ costumam fazer com frequência tal repouso quando chegam em casa, isto se deve ao fato das funcionárias realizarem uma dupla jornada de trabalho (atividades domésticas).

Segundo Thomas (1997), o desconforto causado pela insuficiência venosa tende a melhorar com a deambulação, fato este observado neste estudo quando foi perguntado se o fato de caminhar alterava suas queixas: 40 das funcionárias $(75,47 \%)$ disseram que diminuíam. Silva (1999) explica que, durante a deambulação, as válvulas venosas dos membros inferiores operam em associação com a contração muscular, de modo que o sangue movimenta-se em direção centrípeta, impelido pela contração muscular, o que facilita o retorno venoso e a consequente diminuição dos sintomas.

Quando o indivíduo se coloca na posição ortostática, há uma contração mantida da musculatura envolvida (GUIMARÃES, 2006) e, segundo Harbish apud Baú (2002), durante a contração estática, o músculo enrijece e a pressão intramuscular diminui efetivamente o diâmetro dos pequenos vasos, principalmente das vênulas, o que prejudica a pressão sanguínea dos músculos em questão. Desse modo, o retorno venoso das extremidades é prejudicado, pois depende do fechamento e da abertura das válvulas venosas, que só ocorrem quando os músculos acham-se ritmicamente se contraindo e relaxando. Esse prejuízo do retorno venoso provoca a formação de edema.

A presença de edema, após a jornada de trabalho, foi relatada por $40(75,47 \%)$ das funcionárias, no entanto, foi verificado, através da perimetria antes de iniciar a jornada de trabalho, quando o edema ainda não estava presente, e após o seu término, uma variação na circunferência dos membros inferiores em todas as funcionárias avaliadas. Estes resultados estão de acordo com o estudo de Belczak et al. (2004), segundo o qual a perimetria dos membros inferiores também se mostrou aumentada ao final da jornada de trabalho. No entanto, o autor encontrou uma maior variação de circunferência na região da panturrilha direita, ao passo que, no presente estudo, a maior variação média da medida de perimetria se deu na região do tornozelo bilateralmente, não havendo diferença estatística quando comparados os dois membros. Esta diferença entre os dois estudos pode se dar pelo fato de Belczak et al. (2004) terem observado várias categorias profissionais diferentes - e sabe-se que a atividade laboral constitui um dado importante a ser considerado (SILVA, 2002). Além disso, foi evidenciado que a maioria $(84,91 \%)$ das funcionárias do setor de acabamento realizavam transferência de peso constante para alívio do desconforto nos membros inferiores, dividindo a sobrecarga estática para os dois membros.

A primeira manifestação objetiva da estase venosa nos membros inferiores é o edema, que na fase inicial aparece na metade do dia, apresentando-se de forma mais marcante ao anoitecer (MELLO, 1999). A topografia típica do edema da insuficiência venosa é na perna, predominando no terço inferior (MELLO, 1999; CRAAGER; DZAU, 2002). O edema aumenta progressivamente durante o dia, no pé e na região maleolar (BAREL; MATSUI, 1997; CRAAGER; DZAU, 2002). Fato este também observado neste estudo, onde o edema foi evidenciado principalmente na região do tornozelo.

Ziegler et al. (2003) classificaram a predisposição genética, o sexo feminino, a obesidade e as múltiplas gestações como fatores de risco primários para o desenvolvimento ou o agravamento das doenças venosas de membros inferiores, e as condições do ambiente de trabalho (como o ortostatismo prolongado) como fatores de risco secundários. Já para Sobaszek et al. (1996), os fatores de risco profissionais foram mais determinantes quando comparados com os fatores de risco pessoais. Em relação às disfunções circulatórias observadas no presente estudo, a ocorrência de edema e demais sintomas da doença venosa de membros inferiores parece estar relacionada com as condições de trabalho apresentadas.

Os resultados obtidos neste estudo sugerem que a permanência prolongada na postura ortostática pode ter influência no desencadeamento e/ou no agravamento de sinais e sintomas referentes aos transtornos circulatórios (varizes, ocorrência de dor, sensação de peso ou cansaço, cãimbras e edema, entre outros) nos membros inferiores, embora a medição dos perímetros não tenha sido sensível para detectar alterações de volume dos membros inferiores ao final das jornadas de trabalho. Contudo, para minimizar a incidência dos sintomas de origem circulatória nos membros inferiores em atividades laborais que exijam a postura ortostática, sugere-se que o posto de trabalho seja adaptado para alternância de posturas e que sejam fornecidas, como equipamento de proteção individual (EPI), meias de compressão elásticas. No entanto, não se pode deixar de ressaltar a importância e a possibilidade de aprofundamento do tema, o que poderia evoluir para o estabelecimento de um protocolo de prevenção e tratamento de doenças venosas em função de atividades laborais realizadas em postos de trabalho que exigem a posição ortostática mantida.

\section{Contribuições de autoria}

Berenguer, F. A.: responsável pelo conteúdo intelectual e científico do artigo, pela aquisição e interpretação dos dados e pela redação do manuscrito. Silva, D. A. L.: responsável pela revisão crítica e pela análise estatística. Carvalho, C. C.: responsável pela concepção, pelo delineamento do estudo e pela revisão crítica. 


\section{Referências}

ALLAERT, F. A.; LEVARDON, M.; VIN, F. Influence de l'obesité sur la maladie veineuse: étude des facteurs concomitants. Phlébologie, Paris, v. 44, n. 2, p. 271280, 1991.

ARAÚJO, T. M. et al. Mal-estar docente: avaliação de condições de trabalho e saúde em uma instituição de ensino superior. Revista Baiana de Saúde Pública, Salvador, v. 29, n. 1, p. 6-21, 2005.

AZIZI, M. A. A. Morfometria das fibras elásticas em colaterais varicosas do sistema de veias safenas. Revista de Angiologia e Cirurgia Vascular, Rio de Janeiro, v. 11, n. 1, 2002. Disponível em: <http:// www.sbacvrj.com.br/paginas/revistas/pdf/2002/01/ mor-fibras-elasticas.pdf $>$. Acesso em: 28 mar. 2008.

BAREL, E. V.; MATSUI, I. A. Hipertensão venosa crônica. In: LANE, J. C.; BELLEN, B. V. O exame do paciente vascular. São Paulo: Byk, 1997. p. 147-152.

BAÚ, L. M. S. Fisioterapia do trabalho: ergonomia, legislação, reabilitação. Curitiba: Clãdosilva, 2002.

BELCZAK, C. E. Q. et al. Influência da atividade diária na volumetria dos membros inferiores medida por perimetria e pela plestimografia de água. Jornal Vascular Brasileiro, São Paulo, v. 3, n. 4, p. 304-310, 2004.

BRASIL. Ministério do Trabalho e do Emprego. Manual de aplicação da Norma Regulamentadora $n^{\circ}$ 17. 2. ed. Brasília: MTE, SIT, 2002.

CRAAGER, M. A.; DZAU, V. J. Doenças vasculares dos membros. In: BRAUNWALD E. et al. Harisson: medicina interna. 15. ed. Rio de Janeiro: Mc Graw Hill, 2002. v. 1, p. 1516-1524.

DEJOURS, C. A loucura do trabalho. 5. ed. São Paulo: Cortez-Oboré, 1992.

FRANÇA, L. H. G.; TAVARES,V. Insuficiência venosa crônica: uma atualização. Jornal Vascular Brasileiro, São Paulo, v. 2, n. 4, p. 319-328, 2003.

GRANDJEAN, E. Manual de ergonomia: adaptando o trabalho ao homem. 4. ed. Porto Alegre: Artes Médicas, 1998.

GUIMARÃES, L. B. M. Avaliação do Trabalho Físico. In: GUIMARÃES, L. B. M. Ergonomia de produto: antropometria, fisiologia e biomecânica. 5. ed. Porto Alegre: FEENG, 2006. v. 1.

HANSEN, L.; WINKEL, J.; JORGENSEN, K. Significance of mat and shoe softness during prolonged work in upright position: based on measurements of low back muscle EMG, foot volume changes, discomfort and ground force reactions. Applied Ergonomics, United Kingdom, v. 29, p. 217224, 1998.

JORGE, M. C. T. C. A Postura de trabalho em pé: um estudo de trabalhadores lojistas. 2003. $178 \mathrm{f}$.
Florianópolis. Dissertação (Mestrado em Engenharia de Produção)-Programa de Pós-Graduação em Engenharia da Produção, Universidade Federal de Santa Catarina, Florianópolis, 2003.

JÚNIOR, S. V. Comparação da carga postural dos trabalhadores de duas ilhas de atendimento de uma loja de departamento. 2004. 91 f. Porto Alegre. Dissertação (Mestrado em Engenharia de Produção)Programa de Pós-Graduação em Engenharia da Produção, Univerisdade Federal do Rio Grande do Sul, Porto Alegre, 2004.

KRIJNEN, R. M. A. et al. Venous insufficiency in male workers with a standing profession. Part 1. Dermatology, v. 194, p. 111-120, 1997.

LUZ, C. M. O trabalho na produção de refeição e as doenças venosas de membros inferiores. 2006. 235 f. Florianópolis. Dissertação (Mestrado em Nutrição)-Programa de Pós-Graduação em Nutrição, Universidade Federal de Santa Catarina, Florianópolis, 2006.

MELLO, N. A. Síndrome da insuficiência venosa crônica. In: MELLO, N. A. Síndromes vasculares. São Paulo: Byk, 1999. p. 210-233.

PARISELLE, E. et al. Etude épidemiologique des troubles veineux des members inférieurs selon la posture de travail: a propos de 2.985 dossiers. Archives des Maladies Profissionnelles, v. 54, p. 60-61, Mar. 1992.

PERRIN, M.; GUEX, J. J. Edema and leg volume: methods of assessment. Angiology. n. 51, p. 9-12, 2000 .

PITTA, G. B. B.; FONSECA, F. P; SANTOS, A. D. Varizes do membro inferiores. In: Angiologia e cirurgia vascular: guia ilustrado. Maceió: Uncisal/ECMAL \& LAVA; 2003. Disponível em: <http://www.lava.med.br/livro>. Acesso em: 20 jan. 2008.

RENNER, J. S. Custos posturais no posicionamento em pé, em pé/sentado e sentado nos postos de trabalho de costura na indústria calçadista. 2002. Porto Alegre. 128 f. Dissertação (Mestrado em Engenharia de Produção)-Programa de Pós-Graduação em Engenharia da Produção, Universidade Federal do Rio Grande do Sul, Porto Alegre, 2002.

SILVA, M. J. C. Insuficiência venosa crônica: diagnóstico e tratamento clínico. In: MAFFEI, F. H. A. et al. Doenças vasculares periféricas. 2. ed., v. 2. Rio de Janeiro: Medsi, 2002. p. 1591-1601.

SILVA, M. R. Retorno venoso e débito cardíaco. In: AIRES, M. M. Fisiologia. 2. ed. Rio de Janeiro: Guanabara Koogan, 1999. p. 422-433. 
SOBASZEK, A. et al. Insuffisance veineuse chronique des membres inférieurs en entreprise: enquête réalisée auprès de trois populations de salariés français. Archives des Maladies Profissionnelles, v. 57, n. 3, p. 157-167, 1996.

THOMAZ, J. B. Propedêutica venosa não invasiva e invasiva. In: THOMAZ, J. B; HERDY, C. D. C.

Fundamentos da cirurgia vascular e angiologia. São Paulo: Byk, 1997. p. 48-75.
ZHANG, L.; DRURY, C. G; WOLLEY, S. M.

Constrained standing: evaluating the foot/floor interface. Ergonomics, United Kingdom, v. 34, n. 2, p. 175-192, 1991.

ZIEGLER, S. et al. High prevalence of chronic venous disease in hospital employees. Wiener Klinische Wochenschrift, v. 115, n. 15-16, p. 575-579, Sep. 2003. 